KnapP, M., Cambridge, P., Thomason, C. et al (1992) Care in the Community: Challenge \& Demonstration. Aldershot: Avebury.

TAPS (1993) TAPS research on the evaluation of hospital closure. British Journal of Psychiatry, Supplement 19.
Tomkins, S. (1969) Personality theory and social science. In Interdisciplinary Relationships in the Social Sciences (C. M. Sherif and C. W. Sherll). Chicago: Aldine.

A full list of references is available on request to Professor Huxley.

\title{
Social services response to psychiatric emergencies
}

\author{
Peter Huxley, Professor of Psychiatric Social Work; and Michael Kerfoot, Senior \\ Lecturer in Psychiatric Social Work, University of Manchester, Manchester M13 9PL
}

This paper reports the results of a nationwide survey of social services responses to psychiatric emergencies. The survey is a companion to that by Johnson \& Thornicroft (1991) (J\&T) who reviewed the emergency service options available in psychiatry, including the emergency clinic, general hospital services, the emergency ward, acute day hospital and crisis intervention and residential services, as well as considering the role of sectorised services, and community mental health centres (CMHCs). For the purposes of our respective surveys of health and social services in England and Wales, we have defined a psychiatric emergency as "occurring when someone (patient, friend, relative or professional) requests urgent intervention on behalf of someone in the community who is suffering from a mental disorder".

\section{The study}

The data for the present survey were obtained from a postal questionnaire which was sent to every social services department in England and Wales. The research committee of the Association of Directors of Social Services endorsed the study, and a letter indicating that this was the case was sent with the questionnaire.

\section{Findings}

Sample size. Eighty-two authorities replied (out of 117 authorities in England and Wales), an overall rate of $70 \%$. The overall figure would be $77 \%$ but for the relatively lower return from London, in particular Inner London authorities.

Use of hospitals for emergency admissions. London boroughs and metro/cities are more likely than counties to be working with one district general hospital (DGH) or mental hospital only. Most social services departments (SSDs) have to respond to both mental hospital and DGH units, whereas only $22 \%$ of J\&T's respondents provided both forms of care.

Accident and emergency units. It is unusual, in London and the metro/cities for a local authority to relate to more than one accident and emergency (A\&E) department for psychiatric emergencies. Nearly $60 \%$ of counties have to relate to two or more A\&E departments, and $10 \%$ of metro/cities and $18 \%$ of counties deal with four or more. The distribution is, however, not significantly different between London boroughs, counties and metro/cites.

Community mental health centres. CMHCs which provide psychiatric emergency services for local authorities are much more common (significantly so) in counties; $77 \%$ of counties receive this service, compared with about $40 \%$ of London boroughs and metro/cities.

Emergency help lines. These are, according to our respondents, absent from $68 \%$ of authorities. We believe respondents have interpreted this question narrowly and have therefore failed to include nationally distributed organisations such as Samaritans and, perhaps, more locally based 'lifeline' phone-in services. 
Sectorised services. Twenty-five authorities $(31 \%)$ did not work with any sectorised health districts; $34 \%$ worked with one, and $35 \%$ worked with more than one. The largest number was six. J\&T's figure was $81 \%$ of districts with sectorisation, and during office hours $84 \%$ of the sectorised districts provided emergency services.

Self referrals for psychiatric emergencies. Approximately two-thirds of the sample have no self-referral system for psychiatric emergencies. The situation is similar in all types of local authority.

Availability of medical services. Psychiatrists are readily available in most authorities during the day and 79 of the 82 authorities $(96 \%)$ do not have major difficulties. The problems of availability are more substantial outside of office hours, and 11 of the 18 London boroughs have serious problems, compared with only one metropolitan borough, and five counties. Of the 82 authorities, $65(79 \%)$ do not have major difficulties. Substantial problems with GPs' availability during the day are absent in 77 of the 82 authorities $(94 \%)$ and 70 of the 82 authorities $(85 \%)$ do not have major difficulties with availability at night.

The provision of residential alternatives. Emergency accommodation is provided in 22 authorities $(30 \%$ of those responding to this question; $27 \%$ of the total sample). In 18 this is within pre-existing residential accommodation and only two are special emergency schemes. All save one are available on a 24 hour basis. Ninety per cent of the services operate from a pre-existing residential establishment rather than a special scheme. The provision of emergency accommodation is less frequent in London $(11 \% \mathrm{cf} 33 \%$ in the metro/cities and $30 \%$ in the counties (a nonsignificant tendency). The whole staff receive training in only three of the 21 schemes which provided information ( $14 \%)$.

Alternatives to admission. The most frequently cited (22 times; $39 \%$ of responses; $27 \%$ of the sample) was 'staff visits', and the second most frequent was the use of a hostel (15 times; $27 \%$ of responses; $18 \%$ of the sample). Day centres, hostels and staff visits, when aggregated, are used by exactly half of the sample as alternatives to admission. The provision of alternatives is not related to type of authority or region.

Crisis teams. Only seven authorities provided a 'crisis team' service. No counties have such teams, presumably because they could not be cost-effective in terms of deployment and rapid response.

Deliberate self harm services. London boroughs rely much more than other authorities on non-hospital based social workers to undertake deliberate self harm (DSH) assessments. The social work services provided for DSH patients are primarily 'daytime' services and do not extend outside of normal office hours.

Speed of response to a request for assessment. If a response has not been achieved within the first two hours then there is likely to be quite a delay in getting a response after that. The quickest responses for all categories come from the county areas, closely followed by the metropolitan boroughs. The London boroughs are noticeably slower to respond to requests.

The provision of approved social workers ( $A S W S$ ). The number of ASWs in the 82 authorities is 3463 . The mean number of active ASWs is $\mathbf{4 0}$ (range 9 to 139). The mean population served per ASW is 11,575 (range 1600 to 21,667 ). The mean number of ASWs per 100,000 population is 11.4 (range 4.6 per 100,000 to 62.5 ASWs per 100,000 ). There is a significant association between the type of local authority and the mean number of active ASWs employed. The same is the case for the regional figures, where there is a three-fold variation in the mean number of active ASWs. The number of ASWs is decided on the basis of the number of teams (specialist or generic) in the department in $44 \%$ of cases, by the demands of the emergency duty rota in $20 \%$ and workload data in a further $14 \%$. Only ten of the respondents $(13 \%)$ calculated the number from the population of the authority. About $8 \%$ of the respondents said that they had no idea how the figure was calculated. Fifty-five per cent of ASWs are located in 'generic' settings, $45 \%$ in 'specialist'. Caseloads are remarkably uniform across social work teams (with means of 27 and medians of 26 , and a range of from 10 to 50 cases, for ASWs in all generic and specialist settings). Of the 70 authorities which replied, $40 \%$ felt that they had the right number of ASWs, $17(24 \%)$ had too many and $25(36 \%)$ had too few. The main reasons given for the shortfall were personnel changes $(20 \%)$ and an increase in child care work $(10 \%)$. There were no statistically significant differences between London and the rest of the country in terms of either caseload size or having too many or too few ASWs, although counties were twice as likely $(n=10 ; 37 \%)$ as London authorities $(n=3 ; 18 \%)$ to report too many ASWs and London authorities more likely to report too few $(n=7 ; 41 \%)$ than counties $(n=8 ; 30 \%)$ but less likely to report too few than metropolitan boroughs $(n=13 ; 50 \%)$

Assessments for compulsory admission. The mean number of assessments per authority is 225.48 (range 54 to 534). The total number of assessments in the 12 months covered by this survey was 10,372 . The rate 
of requests per 100,000 show the great difference in requests in London, and in particular in Inner London. Without the London influence there is no difference between individual counties or metro/ cities, or the regions in their use of sections, with the one exception of counties' greater use of section 3. Requests for the use of emergency powers under the Mental Health Act (Section 4) is lower than previously reported $(7.7 \%$ of all requests) and the use of treatment orders (section 3 ) is higher $(34.6 \%$ ).

Section 136. Just under half of the authorities say that they have some difficulty over the use of Section 136. The two major difficulties which emerge relate to (i) unclear policy or an absence of policy, and (ii) problems with the police.

Additional services. Perhaps the most inadequate features of these other services is the lack of availability and the poor quality of the available translation and ethnic minority worker services. Child care, learning disability, police escorts and ambulances appear to be quite widely and well provided.

Overall rating of $A S W$ service. The most common problems were too much work or demand and a lack of resources, followed by problems with coordination of services or the isolation of specialist workers, and the lack of opportunity to practise regularly. Inter-disciplinary problems were relatively rare ( $4 \%$ of respondents). The best aspects were the benefits of specialism $(28 \% ; 22 / 80)$ and the professionalism associated with it $(23 \% ; 19 / 80)$. The other main items mentioned were availability and responsiveness to emergencies $(23 \% ; 18 / 80)$, good working relations with other disciplines $(11 \% ; 9 / 80)$ and good training $(11 \% ; 9 / 80)$.

$A S W$ training. Consortium training is the most common method employed for ASW training. Seventyfour authorities responded, and $62 \%$ use a consortium approach, ten (14\%) share their ASW training with one other authority and $18(24 \%)$ provide their own. Nine of the 18 which provide their own are counties, but there is no significant relationship between type of authority and type of ASW training. Specific sessions on crisis intervention are offered in 57 (out of 70 ) authorities $(81 \%$ ). Only $16 \%$ $(9 / 58)$ offer teaching by a team from a specific crisis intervention service such as the ones at Coventry or Tower Hamlets.

$S S D$ responses to psychiatric emergencies involving young people. These show a fairly heavy reliance upon traditional child care resources, and in only one quarter of cases was a health related service suggested as an appropriate response, in spite of the fact that $68 \%$ of health authorities were reported as having emergency services available.
Caring for people. Authorities were asked to consider how their services for psychiatric emergencies would be affected by the changes outlined in the White Paper Caring for People. Of those responding, $40 \%$ thought that they would be unaffected.

Service quality. Using relevant variables the authorities can be grouped into high quality $(17 \%)$, poor quality services $(22 \%)$ and the rest $(61 \%)$. High quality is as likely (not significantly different) in each type of authority: London $11 \%$; metro/cities $17 \%$ and county $21 \%$. Using these and additional variables related to other agencies produces a 'joint quality' variable. Having to relate to a larger number of DGH units tended to be associated with a poorer quality joint service. Relating to a larger number of psychiatric hospitals was associated with a rather better quality of joint service. However, this result may be due to the type of local authority, because joint quality services are more likely to be good or moderate in counties and poor in London. Poor quality joint services tend to have no sectorised services within them, and the higher quality joint services tend to relate to more sectorised services. This association is also possibly due to the type of local authority because the type of local authority is related to sector. None or one sectorised service are most common in metro/cities, and more than one sector is most common in counties. Coterminosity is also related to quality. Poor quality joint services tended to be in non-coterminous authorities.

\section{Comment}

A number of the results are similar to those of Johnson \& Thornicroft's survey of health districts. Among these are comparable rates for sectorised services in the UK, and the location and availability of emergency services provided by CMHCs. We also found that the frequency of serious difficulties in the availability of doctors during the working day and out of hours was low. Our results differed in respect of the number of authorities which have to work with both DGH units and mental hospitals in respect of psychiatric emergencies; $22 \%$ of health authorities and $48 \%$ of local authorities work to both. We also found similar rates of provision of residential services outside hospital, but we differed in our interpretation of the figure of between one quarter and one third of local authorities making this provision; we regarded this as fairly substantial.

The results reveal a pattern of social service development which is moving from reliance upon psychiatric and mental hospital provision to one which is increasingly based upon sectorised service provision and the availability of CMHCs. In a number of county authorities the CMHC is becoming the locus of emergency services during the day. Counties tend 
to have more rapid response rates to emergency requests, which must, we feel, be related to their continual preoccupation with organising their services to take account of geography and population dispersal. No county in the present study employed a crisis intervention team (CIT) to meet requests for emergency services. Crisis teams are almost exclusively provided by boroughs. A number of authorities still have more ASWs who are approved than active. This may be the result of their blanketing in all members of staff in the early days of the operation of the Act. In some cases the authority still aims to approve as many staff as possible, but many have now found the appropriate level. London boroughs still have nearly $10 \%$ more ASWs approved than active, double the number of inactive ASWs in other types of authority. The range in the number of active ASWs seems to be unjustifiable, even if a case can be made out for London boroughs and some inner cities in other parts of the country having higher rates to respond to the greater frequency of emergency requests produced by such areas.

The number of additional services upon which the ASW can draw was unevenly distributed with very few authorities providing an adequate service over the whole authority. Of most concern is the lack of availability of translation services and ethnic minority workers. Inadequate escort services and other forms of transport are also common, especially in rural areas.

Finally, it appears that joint work with health, facilitated by a sectorised health service, in a coterminous authority, provides the best chance of a quality outcome for clients of psychiatric emergency services.

\section{Acknowledgements}

The authors wish to acknowledge financial support for the project from the Department of Health, endorsement by the Association of Directors of Social Services Research Committee, and the assistance of the social services managers who took part.

\section{References}

Johnson, S. \& ThornicrofT, G. (1991) Psychiatric Emergency Services in England and Wales. Report of a Study Commissioned by the Department of Health. MRC Social \& Community Psychiatry Unit, Institute of Psychiatry, London.

\section{Defeat Depression Campaign}

The Defeat Depression Campaign launched, on 28 April 1993, the first of its leaflets for the general public, on Depression in the Elderly. This is the first in the series of leaflets which the Campaign, in association with the College's Public Education Committee, hopes to release twice a year. Individual copies of the leaflet are available from the College on receipt of a stamped addressed envelope. 